Open dorsal dislocation of the PIP joint is an infrequent case of orthopedic emergencies. Such injuries can be treated safely in the emergency department by closed reduction and extensor splinting until a definite treatment plan is made by an orthopedic hand surgeon. The complications must be taken into consideration. Early active motion leads to significantly superior results in the range of motion than static splinting, because prolonged immobilization may result in flexion contracture.

Prehosp Disaster Med 2011;26(Suppl. 1):s155-s156

doi:10.1017/S1049023X11005061

(P2-63) An Evaluation of 57 Tick Bite Cases M. Ortatatli, ${ }^{1}$ R. Gumral, ${ }^{2}$ H. Uckardes, ${ }^{3}$ M. Eroglu, ${ }^{4}$ L. Kenar, ${ }^{1}$ Y.E. Eyi, ${ }^{4}$ I. Arziman,${ }^{4}$ M. Durusu ${ }^{4}$

1. Department of Medical Cbrn Defense, Etlik - Ankara, Turkey

2. Department of Microbiology, Ankara, Turkey

3. Erzurum, Turkey

4. Department of Emergency Medicine, Etlik - Ankara, Turkey

Crimean-Congo Hemorrhagic Fever (CCHF) is a fatal zoonotic viral infection. The agent belongs to the Nairovirus of the Bunyaviridae species. The virus naturally recycles in vector-vertebrate-vector. This study aimed to evaluate cases of tick bites admitted to Infectious Diseases and Emergency Departments in 2008, and to develop management recommendations of such cases. Fifty-seven patients who admitted to a hospital due to tick bites in 2008 were included in the study. A 10-day clinical follow-up was performed to assess for symptoms including fever, fatigue, abdominal pain, headache, nausea/vomiting, diarrhea, disseminated somatic pain, and other hemorrhagic signs. During this period, laboratory analyzes, including white blood cells, thrombocytes, aspartate aminotransferase, alanine aminotransferase, gamma-glutamyl transferase, lactate dehydrogenase, creatinine phosphokinase $(\mathrm{CK})$, and pentylenetetrazol were performed. Personal data of the patients, location of the bite, and the removal of the tick were recorded.

Results: Of the 57 patients, 37\% were from the city, and 63\% were from rural areas. The tick was removed by health staff in 25 (44\%) of the cases. The bites occurred on body areas including the head/neck, trunk, upper extremities, and lower extremities in $14 \%, 24 \%, 27 \%$, and $13 \%$ of the cases, respectively. During the follow-up period, none of the patients exhibited any of the signs or symptoms listed above. Laboratory tests did not reveal any abnormalities, except for high levels of CK in 15 patients. Thus, 57 cases did not develop CCHF.

Discussion and Conclusion: Since 2002, CCHF has caused an increased mortality in Turkey, and has resulted in high anxiety and concern among the Turkish public regarding tick bites. This has resulted in a rise in the number of patients admitting to emergency departments with tick bites. Due to CCHF's incubation period, patients with tick bites should be evaluated for 10 days using a multidisciplinary approach involving both clinical and laboratory evaluations in order to prevent the unnecessary administration of ribavirine.

Prehosp Disaster Med 2011;26(Suppl. 1):s156

doi:10.1017/S1049023X11005073
(P2-64) Pattern and Factors Associated with Violent Incidents in the Emergency Department of a Level-1 Trauma Center

K. Sharma, S. Bhoi, S. Chauban

Emergency Medicine, New Delhi, India

Background: Violent incidents (VI) in emergency departments (EDs) are under-reported concerns for emergency care providers (ECP). There are limited data from EDs in India.

Objective: This is a study of pattern and factors associated with VIs in the ED of a Level-1 Trauma Center.

Methods: A qualitative survey questionnaire was distributed to $42 \mathrm{ECPs}$ who worked in the ED of the All India Institute of Medical Sciences. Responses of ECPs were compiled and analyzed.

Results: A total of $78.6 \%$ of nurses, $19.1 \%$ doctors, and $2.4 \%$ registration clerks participated in the study. A total of $54.8 \%$ were female. A total of $85.7 \%$ had five years of work experience in the ED. A total of $59.5 \%$ witnessed and experienced verbal abuse; $19.04 \%$ witnessed and experienced physical abuse. The remaining experienced and witnessed verbal abuse as well as physical abuse. A total of $57.1 \%$ had experienced VI multiple times in last six months. Of these, $88.2 \%$ faced the incident between $8 \mathrm{pm}$ and $8 \mathrm{am}$. The ECP reported the incident to hospital authority's $54.8 \%$ of the time, $19.1 \%$ reported it to the police, and $14.29 \%$ did not report, while $7.1 \%$ reported to police and hospital administration. Eighty-five percent experienced insecurity \& emotional disturbance. Patient-related factors included intoxication and anxiety. Healthcare system-related factors included delay in investigations, non-availability of beds, overcrowding of $\mathrm{ED}$, and lack of staff. A total of $47.7 \%$ of participants felt that there was improper communication between healthcare workers and $40.1 \%$ felt that non-availability of senior doctor were the system deficiency factors. A total of $16.6 \%$ of ECPs had formal training regarding communication skills, and $11.9 \%$ had grief counseling. All respondents felt that steps should be taken to decrease and mitigate the VIs in the ED.

Conclusions: Verbal and physical abuse of emergency care workers is common.

Prehosp Disaster Med 2011;26(Suppl. 1):s156

doi:10.1017/S1049023X11005085

(P2-65) Perception of Emergency Care Providers Toward the Implementation of an Electronic Medical Record System in the Emergency Department of a Level-1 Trauma Center

S. Chauban, ${ }^{1}$ S. Bhoi, ${ }^{1}$ D.T. Sinha,${ }^{2}$ M. Rodha ${ }^{2}$ G. Adbikari, ${ }^{2}$ G. Sharma,${ }^{2}$ K. Sharma, ${ }^{2}$ R. Kumar ${ }^{2}$

1. Department of Emergency Medicine, Trauma Centre, India

2. Emergency Medicine, New Delhi, India

Background: Manual documentation has an inherent problem of improper communication, manipulation, and validity. An electronic medical record (EMR) is a computerized medical record created in an organization that delivers care, such as a hospital. EMRs tend to be a part of a local, stand-alone, health information system that allows for storage and retrieval.

Objectives: The objective of this study was to assess the perception of emergency care providers toward the implementation 
of an EMR System in the emergency department of a Level-1 Trauma Center.

Methods: A qualitative survey was conducted among consenting doctors and nurses in the emergency department of the All India Institute of Medical Sciences February to October 2010. Data were collected from a sample of 22-eight doctors and 14 nurses. The collection tool was a structured, closed-ended questionnaire of 12 questions based on usability, applicability, and security, of EMR. A Likert scale (LS) was used (1 = worst, 4 = best). Surveys were done on Day 20, Day 45, and after nine months of implementation of. Responses of emergency care providers were compiled and analyzed using SPSS version 16.

Results: Three surveys consisted of 22 participants in each survey. The survey domain of usability improved on Survey $3(\mathrm{LS}=2.57)$, Survey $2(\mathrm{LS}=2.46)$, Survey $1(\mathrm{LS}=2.24)$. Application of EMR improved from Survey 1 to Survey 3. The data regarding perception of security concerns such as manipulation of data, transparency, and accountability were comparable among Survey 1, Survey 2, and Survey 3. Initial satisfaction was strongly associated with perception of usefulness of data mining for research purposes.

Conclusions: Satisfaction with an EMR system at its implementation generally persisted through the first year of use. Implementation plans must include positive reinforcement regarding EMR among emergency care providers.

Prehosp Disaster Med 2011;26(Suppl. 1):s156-s157 doi:10.1017/S1049023X11005097

(P2-66) Experience of 14 Cases Exposed to Hydrazine S.K. Tuncer, M. Durusu, I. Arziman, Y.E. Eyi, A. Bayir, U. Kaldirim, A.O. Yildirim, M. Eryilmaz

Department of Emergency Medicine, Etlik - Ankara, Turkey

Hydrazine, a highly toxic agent is mainly used as a high-energy rocket propellant or reactant in military fuel cells, in nickel plating, in the polymerization of urethane, for removal of halogens from wastewater, as an oxygen scavenger in boiler feedwater to inhibit corrosion, and in photographic development. Shortterm exposure to high levels of Hydrazine may cause irritation of eyes, nose, and throat, headache, nausea, dizziness, pulmonary edema, seizures, and coma. Acute exposure can also damage liver, kidneys, and central nervous system. Dermatitis may develop by skin contact. In this article we aimed to present our experience belongs to 14 cases exposed to Hydrazine. Cases were evaluated retrospectively based on demographic data, exposure type, approximate exposure time, clinical features, lab analyzes and results of follow-up. Cases were all male personnel. Mean age and standard deviation were 30,28 and 6,73 respectively. All cases were exposed to Hydrazine in an open place during the monitorization of aircraft for a couple of seconds. Personnel were presented to feel an odor similar to garlic in their nasopharynx. Retrosternal burning was the preponderant symptom in 6 of the cases. The vital signs and physical examination provided no valuable data. Evaluation of Whole Blood Count, Arterial Blood Gas, Biochemical Parameters, Urine Tests, ECG and Chest Radiograph took place in diagnosis period. Respiration function tests were performed on the 6 of the cases who had respiratory complaints. All tests revealed unremarkable data. All cases were subjected to reevaluation in the end of next 48 hours. No complications were encountered on the next examination. Our cases presented no mortality and complication due to having information about Hydrazine and short-term exposure and exposure in open place. Of personnel working in such places including Hydrazine, having information about Hydrazine, is the leading factor in preventing mortality and complications of Hydrazine.

Prehosp Disaster Med 2011;26(Suppl. 1):s157

doi:10.1017/S1049023X11005103

\section{(P2-67) Torsade De Pointes and Ventricular Fibrillation Accompanying Intracerebral Hemorrhage \\ C. Hsu, ${ }^{1}$ Y. Chen ${ }^{2}$ \\ 1. Emergency, Taipei, Taiwan \\ 2. Department of Emergency Medicine, Taipei, Taiwan}

A 43-year-old patient with well healthy in the past was admitted after a accidental falling down injury. Upon arrival at the Emergent Department, he was unresponsive. (Glasgow coma scale = E1V1M1). The continuous Electrocardiological monitor demonstrated ventricular fibrillation without pulse, and the defibrillation $(360 \mathrm{~J})$ with intravenous Epinephrine $(1 \mathrm{mg})$ push was given. The electrocardiogram (ECG) returned to sinus tachycardia. The endotracheal tube was intubated to keep airway patent and ventilation under the unstable vital sign. Ten minutes later, the polymorphic ventricular tachycardia (torsade de pointes) were recorded by continuous ECG. After defibrillation $(360 \mathrm{~J})$ twice with intravenous Epinephrine (1 mg) and Amiodarone (150 mg), the rhythm returned to normal sinus rhythm, and the vital sign recovered gradually. The laboratory evaluation showed no abnormality. The cranial computed tomography was done thereafter which showed occipital bone fracture with subarachnoid hemorrhage, subdural hemorrhage and epidural hemorrhage (Figure 2, arrows indicated hemorrhage). Clinical study has shown increased sympathetic activity in patients with acute intracranial hemorrhage. The increased level of catecholamines would lead to QT prolongation or hypokalemia, which are the predisposing factors of the development of torsade de pointes. Although, the definitive confirmation of a cause and effect relationship about intracranial hemorrhage and torsade de pointes is still controversial, the life-threatening arrhythmia, including atrioventricular blocks, ventricular tachycardia, and fibrillation, which accompany acute cerebral accidents in patients without cardiac disease is observed in many case reports. In our experience and clinical observation, patients should be constantly monitored after acute cerebral events. Besides, the treating team should be familiar with and well-trained in the diagnosis and treatment of cardiac arrhythmias.

Prehosp Disaster Med 2011;26(Suppl. 1):s157

doi:10.1017/S1049023X11005115

(P2-68) Mental State of Healthcare Workers in a Designated H1N1 Screening Center

E. Seow, G. Hsin kai, A. Vasu

Emergency Department, Singapore, Singapore

Introduction: In Singapore, the H1N1 outbreak lasted 108 days. The study emergency department (ED) was designated as Singapore's H1N1 screening centre for the borders and the 